\title{
Targeting microbubbles with Shiga-Toxin B-subunit
}

\author{
Couture O, Tanter M \\ Institut Langevin Ondes et Images, ESPCI \\ Paris, France
}

\author{
Dransart E, Dehay S, Johannes L \\ Institut Curie \\ Paris, France
}

\begin{abstract}
The targeting moiety (B-subunit) of the Shiga toxin (STxB) is a very potent ligand for the glycolipid Gb3, which is expressed in ovarian, colorectal and breast carcinomas. It is also present on endothelial cells of tumor neovascularization. This study demonstrates the use of $\mathrm{ST} \times \mathrm{B}$ for targeting microbubbles onto cancerous cells.
\end{abstract}

STxB-functionalized microbubbles and biotinylated controls were incubated with colorectal carcinoma HT29 in opticell plates. The culture plates were observed by fluorescence microscopy. The plates were then installed in a water-bath under a $8 \mathrm{MHz}$ transducer array. STxB and control microbubbles were also injected in nude mice with subcutaneous breast tumors, before ultrasound imaging.

FACS analysis demonstrated that STxB was stably associated with the microbubbles. Fluorescence microscopy showed that STxB-functionalized microbubbles adhered favorably to the Gb3 expressing cells, as compared to cells in which Gb3 expression was inhibited. Disruption ultrasonography of the culture plates showed a $12 \mathrm{~dB}$ difference in average backscatter intensity of the surface of Gb3 expressing cells, compared to Gb3-negative cells. An intensity difference of $18 \mathrm{~dB}$ was also observed between cells that were incubated with STxB-functionalized-microbubbles, as compared to unspecific microbubbles. The experiment in mice showed a significant increase in microbubble-signal intensity within gb3-positive tumors after being injected with STxBmicrobubbles.

These in vitro and in vivo experiments showed that STxBfunctionalized microbubbles bind specifically to cells expressing the Gb3 glycolipid. The targeting moieties of toxins are a new group of ligands for microbubbles and have several advantages compared to antibodies and small peptides.

Key words - Molecular imaging, microbubbles, targeted, shiga, glycolipid, gb3

\section{INTRODUCTION}

Since microbubbles have only access to the lumen of blood vessels, molecular imaging with ultrasound requires targets that are expressed specifically on the wall of vascular endothelial cells. Receptors that are overexpressed in tumor neovascularities such as VEGF-R or Integrins have been targeted through antibodies or short peptides attached to the surface of ultrasound contrast agents.

In this study, we present a new type of ligand for targeted ultrasound contrast agents (TUCA): a subunit of the Shiga toxin $[1,2]$. We also propose a new target: the glycolipid gb3. The glycolipid gb3 is overexpressed in various types of tumor cells such as ovarian cancer, breast cancer, myeloma, lymphoma, colon cancer and metastasis [3]. As shown in figure 1, it is also overexpressed in the neovascularity of tumor. As a glycolipid, gb3's generation and cycle are different from receptors and can provide complementary information.

The Shiga toxin, produced by Shigella dysenteria, is a natural ligand to the glycolipid gb3. The B-subunit, which allows the attachment and internalization of the toxin in the cell, has a very high affinity to gb3 and recruits up to 14 glycolipid sites on a cell. The Shiga-toxin-b-subunit (STxB) evolved to induce low immunogenic response. It has been used as a cargo for targeted drug delivery Because of these advantages; STxB could be a powerful ligand for neovascularity targets.

This article describes the development of the gb3-targeted microbubbles (STxB-microbubbles). In particular, the fabrication of the agent, its targeting to cells in-vitro and to tumors in vivo are illustrated.

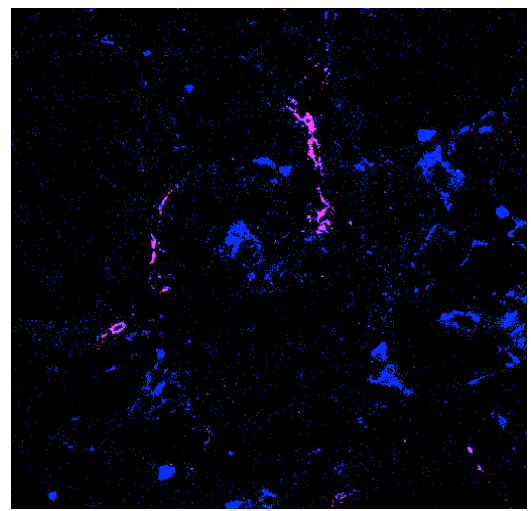

Figure 1: Colocalisation of StxB and neovessels. (Viel et al, Molecular Imaging 2008, [4]) 


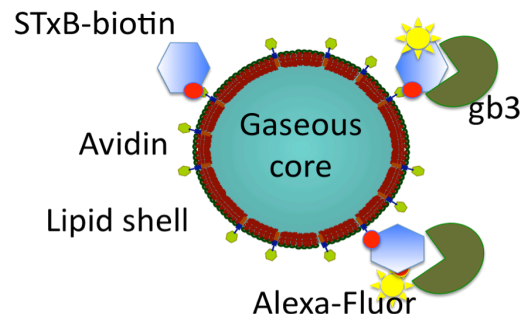

Figure 2: Shiga B-subunit is attached to microbubbles through avidin-biotin complex

\section{MATERIALS AND METHODS}

a) Preparation of the targeted microbubbles

Genetically modified Shiga toxin is combined to biotin on cystein residue, and then to Alexa fluor 488 on primary amines. Avidinated microbubbles (Bracco Research) are prepared following the makers' protocol. Biotinylatedfluorescent-STxB is added to the solution of microbubbles in order to saturate the binding sites of the microbubbles $(3 \mu \mathrm{g}$ in $700 \mu \mathrm{L})$. Control microbubbles are prepared by adding 0.02 $\mu \mathrm{g}$ of biotin in $700 \mu \mathrm{L}$. The microbubbles and the ligand interact for 20 minutes (figure 2). The microbubbles are washed three times by centrifugation before observation under fluorescence microscopy for confirmation of proper binding.

b) Imaging microbubbles on tumor cells in vitro

Targeted microbubbles are combined with human colon adenocarcinoma (HT29) cells. After washing, FACS is performed on the solution and the individual fluorescence of cells (gb3 positive or negative) is measured.

HT29 cells are grown in opticell plates, made of two membranes encapsulating a sterile chamber transparent to both light and ultrasound. After culture, one membrane is covered with HT29 cells (gb3 positive and negative). $250 \mu \mathrm{L}$ of the solution of targeted microbubbles is injected with $12.5 \mathrm{~mL}$ of medium inside the plate. The microbubbles are left to interact with the cells (on top) for 20 minutes. Then, the medium is replaced three times while rocking the plate (cells on the bottom) to remove the unspecific microbubbles.
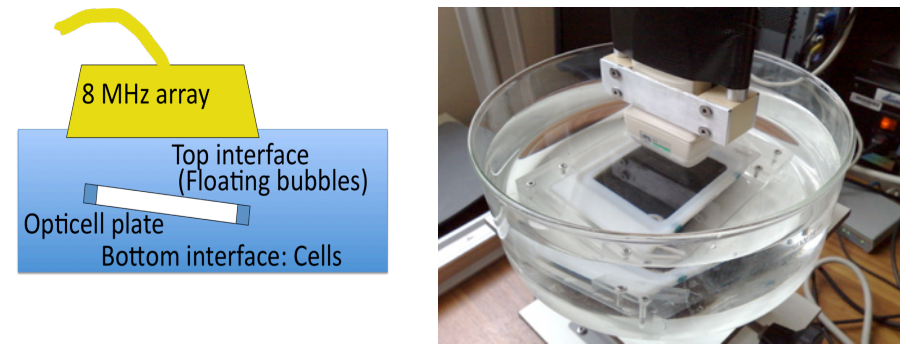

Figure 3: Setup to measure the acoustic reflection of microbubbles attached to cells in an Opticell plate.
Opticell plates are imaged by fluorescence microscopy to observe both microbubbles (Alexa Fluor 488 - green) and the cell membrane (FM dye - red). Then, the plates are transferred into a waterbath under a $8 \mathrm{MHz}$ transducer driven by an ultrasound scanner (Supersonic Imagine, Aix-En-Provence, France). The plate is oriented so that the cells and, eventually, the targeted microbubbles are on the bottom surface (figure 3 ). The unspecific microbubbles left after washing float on the top surface which can be axially resolved from the bottom membrane. Several b-mode images with microbubbledisruption contrast imaging are obtained over the plate. Signal intensity is averaged over the echo of the bottom surface.

c) Imaging microbubbles in tumor in-vivo

Breast cancer cells (BC174) are grown subcutaneously in nude mice. After IP anaesthesia, microbubbles are injected in the retro-orbital sinus of the mouse as a bolus of $100 \mu \mathrm{L}$. Imaging is performed at $8 \mathrm{MHz}$ with a 256-elements array following a plane-wave imaging method. The sequence is modified to add amplitude modulation (odd and even elements) technique to distinguish the bubbles. To prevent the destruction of the microbubbles, imaging is only performed every minute. Imaging is done over the tumor from 5 minutes after the microbubble injection until 15 minutes after. Eight mice were injected with the STxB microbubbles or the Biotinmicrobubbles (control).

\section{RESULTS}

Under fluorescence microscopy (figure 4), the membrane of microbubbles is clearly delineated. Since Alexa Fluor is conjugated to the Shiga-B-subunit directly, STxB consequently functionalizes the microbubbles. As shown on the FACS analysis of figure 5, Gb3-positive cells are bound to more fluorescent microbubbles than the Gb3-negative cells.

Fluorescence imaging of the plate (figure 6) underlines the cell membrane in red and the microbubbles in green. Targeted microbubbles are present in greater numbers on Gb3 expressing cells as compared to the control. There is an average of 1.2 microbubble per Gb3-positive cell as compared to 0.2 per $\mathrm{Gb} 3$-negative cell $(\mathrm{n}=160)$. In $\mathrm{Gb} 3$ positive plates, $80 \%$ of the visible microbubbles are attached to cells while only $25 \%$ of the microbubbles are collocated to cytoplasmic membranes in Gb3-negative cells.

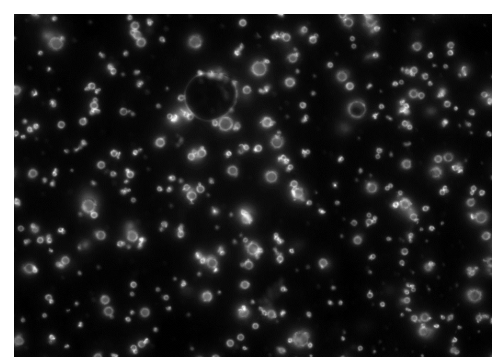

Figure 4: Microbubbles covered with fluorescent-labeled STxB (63X). 
Ultrasound imaging of the bound microbubbles is done in plates at $8 \mathrm{MHz}$. The two membranes can be distinguished on the B-scan. The advantage of these plates is that the unspecific microbubbles float to the top and can be readily eliminated from the ultrasound signal intensity calculation. Ultrasound signal is measured with a disruption sequence, which isolate the bubble-specific signal. In the first pair of controlled testing, the signal from the bottom surface is $12 \mathrm{~dB}$ higher for Gb3 expressing cells compared to negative cells (figure 7). In the second set of controlled testing (figure 8), the microbubbles conjugated with STxB display a signal $18 \mathrm{~dB}$ higher than the unspecific microbubbles. The difference is significant, but depends on the baseline signal of the membrane.

Experiments in vivo are performed on breast cancer implanted subcutaneously in mice. The injection of microbubbles in the retro-orbital sinus, a new technique, was previously tested with unspecific microbubbles. It demonstrated that microbubbles could be easily identified in the organs of the mouse after injection. Using plane-wave imaging technique, the $5 \mathrm{~mm}$ diameter tumors are clearly identifiable as hypoechoic massed under the skin. The green overlay represents the signal from the amplitude modulation sequence after removal of moving specular reflectors. The binding dynamic of the microbubbles was imaged between 5 and 15 minutes after injection. This dynamic showed an important variation in the total number of microbubbles injected at the beginning. However, at the end of the imaging time (15 minutes), the tumor injected with STxB-microbubbles appeared much brighter within its core. Apart from imaging artifacts, the tumors that were injected with biotinmicrobubbles appeared mostly dark. The decrease in internal signal is twice as fast for biotin-microbubbles than STxB microbubbles, demonstrating that the targeted microbubbles are retained in the tumor. For the 8 mice tested (4 shiga, 4 control), the signal from microbubbles appeared reproducibly higher when STxB was conjugated to the agent.
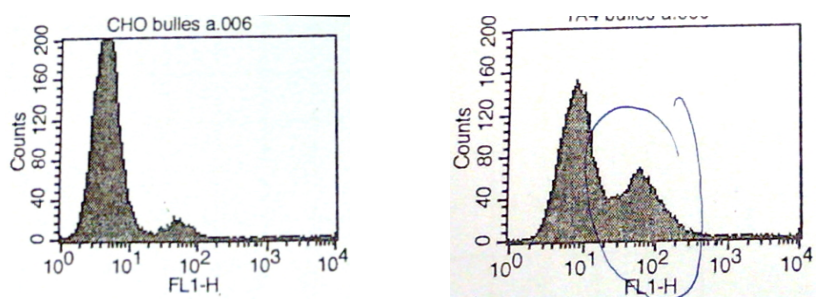

Figure 5: FACS analysis of the binding between the STxB microbubbles and $\mathrm{CHO}$ cells that expresses (right) or not (left) the target Gb3.
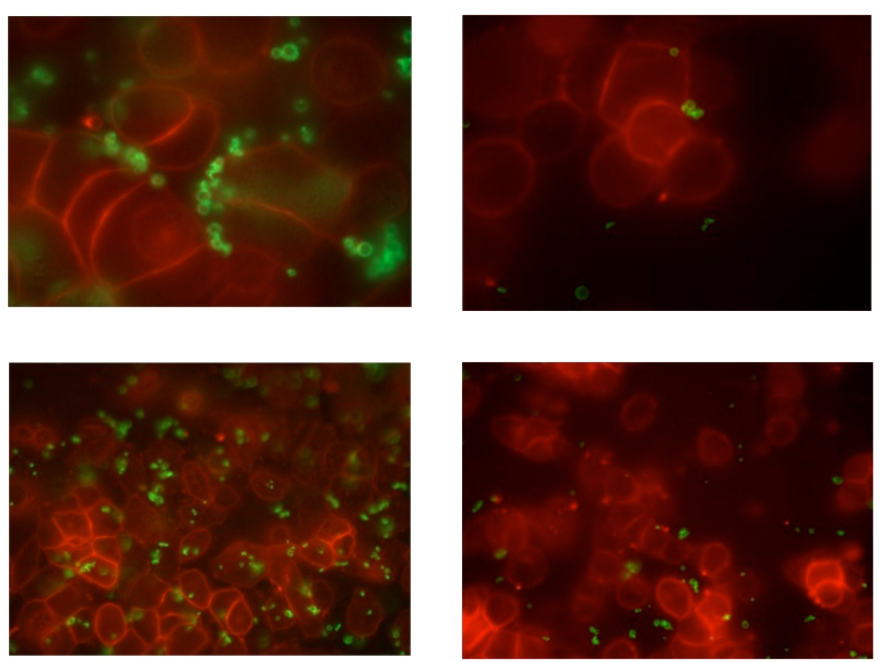

Figure 6: Microbubbles (green) attached to HT29 cells (red FM dye) grown in Opticell plates.

\section{DISCUSSION}

In this study, a new type of ligand and a new target were introduced. Targeted microbubbles were tested, from preparation of the agent to the in-vivo testing.

Fluorescence imaging demonstrated that the Shiga-Toxin-Bsubunit (STxB) was indeed present on the microbubbles. Experiments on FACS and opticell plates, showed that Shiga targeted the microbubbles on the cytoplasmic membrane of Gb3 expressing cells. Bubble-specific signal at the bottom of the plate was significantly higher when they were targeted to Gb3-positive cells as compared to control. Finally, in-vivo experiments demonstrated that the targeted microbubbles accumulated specifically inside the tumor.

Additional experiments should be performed in vivo. Firstly because we introduced a new method of injection which gives rapid and free access to the blood flow, but limits the possibility of catheterization. Consequently, the bolus volume of microbubbles is difficult to control. The number of targeted microbubbles in normal tissue should also be measured to provide normalization for the signal level. Both STxB and control experiments should also be performed on the same mouse to show the accumulation in the same tumor.
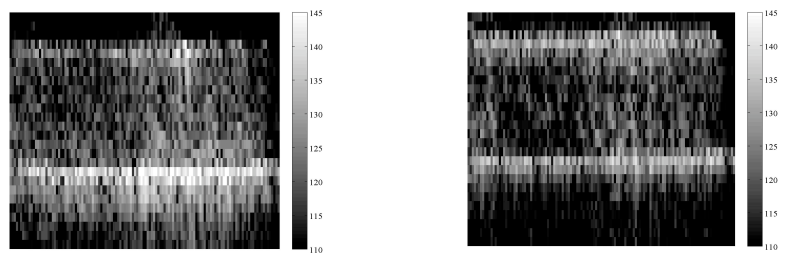

Figure 7: Ultrasound scan of Opticell plate covered with HT29 cells and washed with a microbubble solution. Dual control: STxB microbubbles on $\mathrm{Gb} 3+$ (left) and Gb3- (right) cells. 

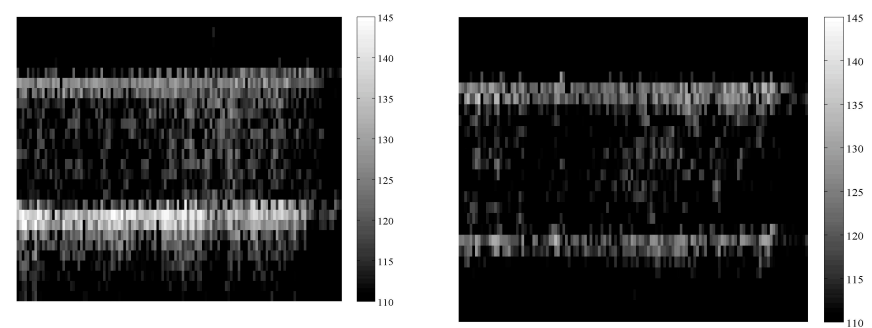

Figure 8: Ultrasound scan of Opticell plate covered with HT29 cells and washed with a microbubble solution. Dual control: STxB (left) and biotin (right) microbubbles on Gb3-positive cells.

Molecular imaging with Shiga-toxin could open new possibilities in angiogenesis imaging. The glycolipid gb3 is not a protein like most receptors and its cycle is an independent property of cancer cells. These new ligands and targets could provide complementary information to VEGF-R, especially after anti-VEGF therapy. The Shiga ligand could also help reduce the immunogenicity of targeted microbubbles when used as targeted ultrasound contrast agents. Glycolipid gb3 has been identified as a possible target for therapy. STxBmicrobubbles could help predict the zone of action of targeted drug delivery with Shiga toxin.

Considering its affinity to Gb3 expressing tumor, STxBmicrobubbles could become a useful tool for molecular imaging, but also the targets for the molecular focusing therapy previously described by our laboratory [5].
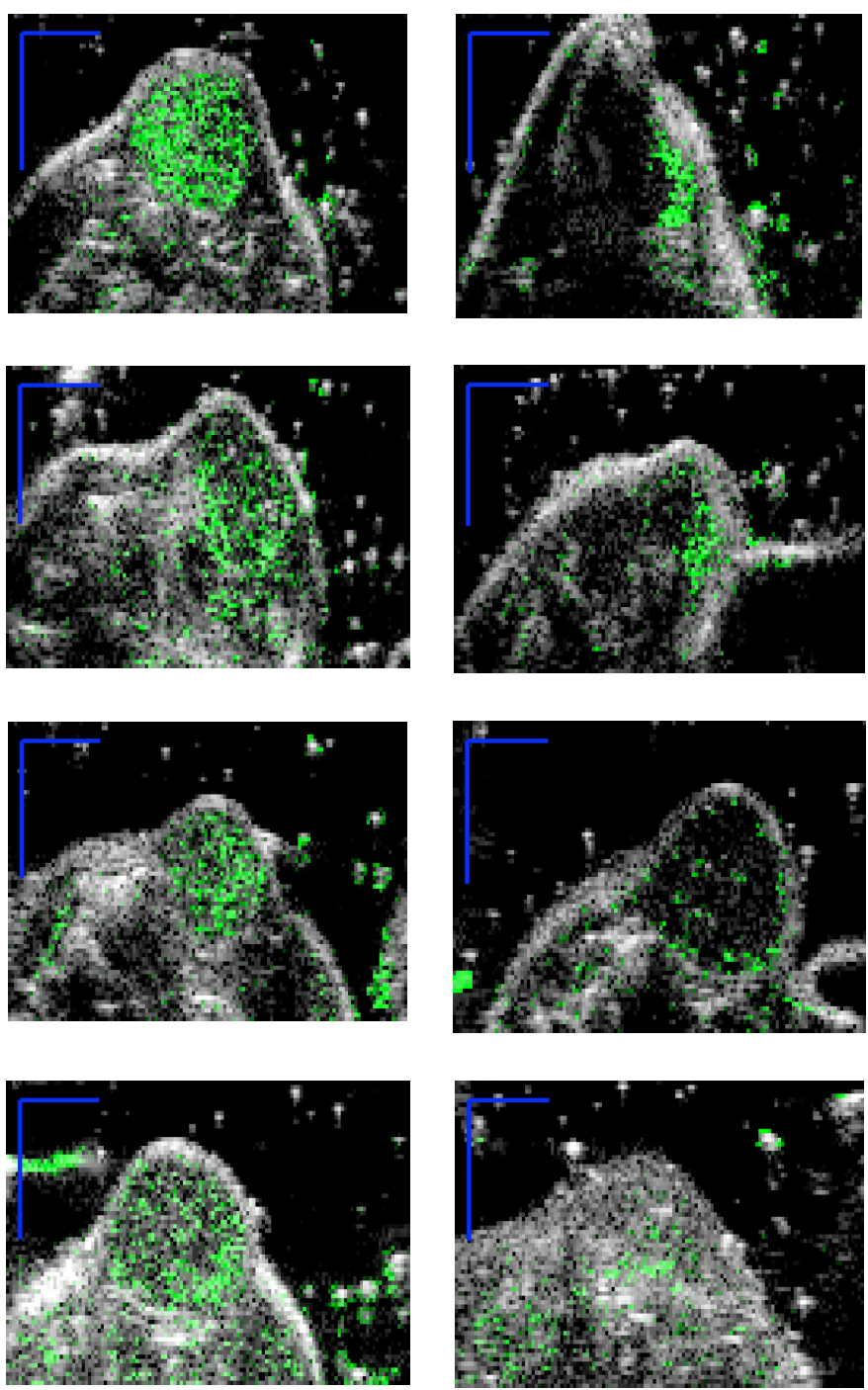

Figure 9: Ultrasound imaging of $\mathrm{BC} 174$, gb3-positive, tumors. The green overlay is the bubble-specific amplitude- modulation signal. The blue scale line is $5 \mathrm{~mm}$ across. Images were obtained on 8 different mice.

\section{REFERENCES}

[1] Johannes L, Decaudin D. Protein toxins: intracellular trafficking for targeted therapy. Gene Therapy 12, 1360-1368, 2005.

[2] Gariepy J. The use of Shiga-like toxin 1 in cancer therapy. Critical reviews in oncology/hematology 39, 990106, 2001.

[3] LaCasse EC, Bray MR et al. Shiga-Like Toxin-1 Receptor on Human Breast cancer, lymphoma and myeloma. Blood 94, 2901-2910, 1999.

[4] Viel T, Dransard E, Nemati F et al. In vivo targeting by the b-subunit of shiga toxin. Molecular imaging 7, 239-247, 2008.

[5] Couture O, Aubry JF, Tanter M, Fink M. Time-reversal focusing of therapeutic ultrasound on targeted microbubbles. Applied physics letters 94, 2009. 